\title{
Le rôle des dépôts de médicaments dans la circulation informelle de médicaments à Madagascar
}

Chiarella Mattern

\section{OpenEdition}

Journals

Édition électronique

URL : https://journals.openedition.org/rfst/1024

DOI : $10.4000 /$ rfst. 1024

ISSN : 2492-3672

Éditeur

Espaces et SOciétés (UMR 6590)

Référence électronique

Chiarella Mattern, «Le rôle des dépôts de médicaments dans la circulation informelle de médicaments à Madagascar », Revue francophone sur la santé et les territoires [En ligne], Les circulations en santé : des produits, des savoirs, des personnes en mouvement, mis en ligne le 10 juin 2021, consulté le 12 juin 2021. URL : http://journals.openedition.org/rfst/1024 ; DOI : https://doi.org/ $10.4000 /$ rfst. 1024

Ce document a été généré automatiquement le 12 juin 2021.

\section{cc) (†) $(2)$}

La Revue francophone sur la santé et les territoires est mise à disposition selon les termes de la Licence Creative Commons Attribution - Pas d'Utilisation Commerciale - Partage dans les Mêmes Conditions 4.0 International. 


\title{
Le rôle des dépôts de médicaments dans la circulation informelle de médicaments à Madagascar
}

\author{
Chiarella Mattern
}

\section{Introduction}

1 La circulation géographique de produits utilisés dans un cadre thérapeutique à différentes échelles spatiales est un phénomène qui tend à s'accroitre depuis plusieurs décennies avec la progression des échanges commerciaux, les facilités de déplacement, les migrations et la diffusion de la biomédecine à l'échelle mondiale. Les produits de santé $e^{1}$ participent aujourd'hui de la mondialisation économique et représentent un marché lucratif difficile à quantifier du fait des circuits et réseaux empruntés qui sont tant formels qu'informels. La circulation des produits en santé peut s'analyser d'un point de vue transfrontalier (Baxerres et al. 2011; Bourdier et al. 2014), la question de la règlementation se pose alors avec acuité. En fonction du degré de surveillance des frontières, les réseaux de vente des médicaments peuvent aussi contourner plus ou moins facilement les autorités de contrôle et faire ainsi émerger un commerce informel du médicament (Quet et al. 2018). La circulation des produits en santé peut se considérer également à l'échelle nationale par l'analyse de l'organisation des circuits de distributions privés, publics et informels ; telle est l'approche adoptée dans le cadre de cet article. Dans nos travaux, nous avons choisi de caractériser ce marché d'« informel " au sens où il relève d'une pratique de vente et d'achat de médicaments se déroulant en dehors d'un cadre formel imposé par l'Etat et par le système de santé biomédical qui prévaut à Madagascar. La formulation « marché informel » est généralement choisie en anthropologie pour désigner ces pratiques (Baxerres, 2015).

2 A Madagascar, les voies d'accès aux médicaments prévues pour les populations sont plurielles. Le système public assure l'approvisionnement en médicaments des pharmacies de centres de santé de base et hôpitaux tandis que le système privé 
approvisionne les pharmacies dans les agglomérations urbaines et les dépôts de médicaments en milieu rural (fivarotam fanafody). Les médicaments pharmaceutiques sont également distribués via les circuits informels, tant en milieu rural qu'en milieu urbain. La répartition des circuits formels laisse de nombreuses zones en proie à une faible couverture en médicaments. A Madagascar, « les problèmes [en termes d'accès aux médicaments] semblaient se situer non au niveau d'une pénurie générale, mais à celui d'une organisation défectueuse à tous les niveaux d'approvisionnement, et de trafics non sanctionnés » (Mestre, 2014).

3 Une des caractéristiques du système pharmaceutique malgache est la présence d'un acteur incontournable dans la distribution, rendant possible l'accès aux médicaments pour les populations vivant en milieu rural : les dépôts de médicaments. Ceux-ci ont été mis en place en 2011 pour pallier le manque de pharmaciens sur l'île et pour répondre aux problèmes d'accessibilités des populations aux médicaments essentiels.

4 Ce présent article rend compte du rôle des dépôts de médicaments dans l'approvisionnement du secteur informel. Partant de l'analyse de l'organisation de la distribution de médicaments à Madagascar, nous décrirons les caractéristiques, mécanismes de fonctionnement et modalités d'approvisionnement de ces dépôts.

Cet article se base sur les résultats de ma thèse, dont les données ont été collectées par trois biais : entretiens semi-directifs, observations directes et relevés des points de ventes informels. Une centaine d'entretiens semi-directifs a été réalisé entre 2013 et 2017 à Madagascar auprès d'acteurs issus des différents secteurs de distribution pharmaceutique. Les observations des contextes de dispensation de médicaments ont été réalisées à Ambohipo ${ }^{2}$ durant les moments de "flottement ", en attendant qu'un vendeur soit disponible pour l'entretien, ou durant les entretiens, lorsque nous étions interrompus par un acheteur, ce qui arrivait fréquemment. Nos observations visaient à comprendre les liens entre vendeurs, et la relation entre le vendeur et l'usager autour de l'acquisition du médicament. Enfin, un relevé non exhaustif des points de vente de médicaments informels a été réalisé au sein des huit marchés hebdomadaires et permanents les plus fréquentés et renommés de la capitale Antananarivo ${ }^{3}$. En complément, des observations et entretiens courts ont été menés avec soixante-dix commerçants à l'étalage (légumes, vêtements, produits de première nécessité (PPN), remèdes traditionnels), des marchands ambulants (vendant des sachets en plastique, des DVD, des produits alimentaires, des remèdes traditionnels) et des épiciers dans l'objectif d'identifier les différentes catégories de vendeurs, et les voies de circulation informelles des médicaments à Antananarivo.

\section{Caractéristiques du système pharmaceutique malgache}

\section{Trois secteurs de distribution}

6 À Madagascar, la distribution de médicaments se fait au travers de trois secteurs : le privé - regroupant les grossistes répartiteurs, les pharmacies d'officine et les dépôts de médicaments, le public représenté aux différents niveaux de la pyramide sanitaire et approvisionnés par la Centrale d'achat SALAMA et le secteur informel. 
7 En termes d'approvisionnement du secteur public, la centrale d'achat SALAMA ${ }^{4}$ est chargée de l'acquisition, du stockage, du contrôle de qualité et de la distribution des médicaments essentiels ${ }^{5}$ génériques ${ }^{6}(\mathrm{MEG})$ et des consommables jusqu'au niveau des hôpitaux et des districts sanitaires (PhaGDis). La SALAMA s'approvisionne en médicaments sous Dénomination Commune Internationale (DCI). Sa mission consiste principalement à assurer la disponibilité permanente en médicaments essentiels génériques de qualité et à prix abordable à tous les échelons de la pyramide sanitaire organisée en quatre niveaux : les Centres de Santé de Base (CSB) niveau 1 et niveau 2 (au nombre de 2563) ; les Centres Hospitaliers de Référence de District (CHRD au nombre de 87) ; les Centres Hospitaliers de Référence Régionale (CHRR au nombre de 16) ; les Centres Hospitaliers Universitaires (CHU au nombre de 22). La SALAMA achemine les commandes auprès des entrepôts PhaGDis, soit les 111 entrepôts des pharmacies de gros du district, selon un calendrier de livraison trimestriel pour les zones accessibles et semestriel pour les zones isolées. Interviennent ensuite les PhaGeCom ${ }^{7}$ (les pharmacies à gestion communautaire), responsables de la récupération des médicaments auprès du PhaGDis. L'approvisionnement et la gestion des médicaments des centres de santé reposent sur le principe de la participation financière des usagers. Les sommes recouvrées sur les médicaments sont utilisées pour le fonctionnement des formations sanitaires (indemnités pour le paiement du gardien du centre de santé, frais de transport des médicaments du niveau district à l'échelon inférieur, etc.) et en priorité pour la reconstitution des stocks de médicaments.

8 Le réseau de distribution du secteur privé commercial à but lucratif ${ }^{8}$ comprend les grossistes importateurs répartiteurs, les pharmacies d'officine et les dépôts pharmaceutiques. On y dénombre au moment de l'enquête 34 grossistes répartiteurs concurrents. 7 d'entre eux détiennent $80 \%$ du marché pharmaceutique malgache privé. Le volume d'activité des 27 grossistes restants est faible et leur impact sur le marché est limité. Madagascar compte 1672 dépôts de médicaments et 219 pharmacies d'officines privées dont près de la moitié (86) se trouve à Antananarivo. Par ailleurs, le secteur privé non lucratif détient une place importante en matière de prestations des services à Madagascar puisque selon les données disponibles, le secteur santé de l'Eglise Catholique (l'EKAR) dispose de 143 centres de santé, celui de l'Eglise Protestante (SAF/ FJKM) comporte 32 centres de santé. Ces centres offrent un ensemble de soins préventifs et curatifs en priorisant la santé maternelle et infantile (consultations prénatales, nutrition, planning familial et vaccination).

Quant au secteur de distribution informelle, il participe à Madagascar plus largement à ce que certains auteurs nomment le Secteur Informel de la Santé (SIS) (Bloom et al., 2011 ; Nsarhaza, 1997 ; Sudhinaraset et al., 2013). Le terme « informel» englobe une grande variété de dispensateurs de soins et de produits pharmaceutiques, pratiquant régulièrement ou occasionnellement des activités pour lesquelles ils ne possèdent pas la certification médicale requise, selon les autorités sanitaires et / ou la législation couvrant la fourniture de services de santé. Selon Nsarhaza, outre la distribution de médicaments, les réponses informelles en matière de santé couvrent plusieurs réalités : soins à domicile, corruption dans les services médicaux publics, alimentation et nutrition, planification familiale, solidarité face aux risques liés à la santé (1998).

10 Cet ensemble de secteurs constitue le système pharmaceutique malgache, chapeauté par deux organes de règlementation. Le premier est l'Autorité de Règlementation Pharmaceutique (ARP), appelée Agence du médicament de Madagascar (AGMED) et 
créée en 1998. L'AGMED agit sous tutelle du Secrétariat Général de la Santé. Cet organe s'acquitte d'un certain nombre de fonctions relevant du domaine pharmaceutique : dispensation de l'autorisation de mise sur le marché (AMM), inspection pharmaceutique, contrôle de qualité et pharmacovigilance. L'AGMED délivre les autorisations aux officines pharmaceutiques et en assure la régulation. La Direction de la Pharmacie, des Laboratoires et de la Médecine Traditionnelle (DPLMT) constitue l'autre organe de régulation. Placée sous la tutelle de la Direction Générale de la Santé, c'est cette entité qui élabore la Politique Pharmaceutique. Elle est en charge de la délivrance des autorisations et la gestion des dépôts de médicaments et de l'élaboration de la liste des médicaments autorisés à la vente dans ces dépôts.

\section{Un nombre particulièrement faible de pharmacies d'officine}

11 Selon le Code de la Santé (2011), Madagascar a hérité du système qui attribue le monopole de la distribution du médicament au pharmacien, faisant de celui-ci l'acteur majeur de la distribution. Entre 2006 et 2016, 225 pharmaciens ont été formés au niveau national. Avant la mise en place du programme de formation en pharmacie à l'Université d'Antananarivo, les candidats se formaient en Europe, en Chine ou en exURSS $^{9}$. Le ratio Pharmacien par habitant est estimé à $1 / 78000$ en $2014^{10}$ contre 1 pharmacien pour 20000 habitants tels que recommandé par l'OMS. Aujourd'hui plusieurs écoles de pharmacie sont ouvertes sur l'ile, mais les pharmaciens nouvellement formés choisissent pour la plupart d'exercer en ville, refusant les opportunités de travail en province et « d'aller travailler en brousse » ou « d'être envoyés en bout de piste ».

12 A Madagascar, la localisation des pharmacies d'officines n'est pas soumise à des critères géo-démographiques, cela signifie donc qu'il existe de fortes disparités de localisation d'officines sur l'île. L'autorisation d'exploitation est délivrée aux pharmaciens par l'AGMED. Le Code de la Santé (article 155) prévoit que les pharmacies d'officines soient tenues par des pharmaciens. La substitution d'un médicament princeps par son générique équivalent existe mais n'est pas réglementée. Ainsi, les pharmaciens et les dépositaires proposent princeps et générique au client qui fait son choix en fonction de son budget.

\section{Des structures pharmaceutiques inégales}

13 Derrière la dénomination " pharmacie » opèrent des structures inégales quant à l'offre de produits pharmaceutiques. La plupart d'entre elles vendent des produits cosmétiques, des produits à usage des ménages (lait maternisé, couches, etc.) et des médicaments conditionnés ${ }^{11}$. Certaines officines vendent également des Remèdes Traditionnels Améliorés (RTA).

\section{Mise en place des dépôts de médicaments}

14 La dernière caractéristique majeure du système pharmaceutique malgache est la mise en place de dépôt de médicaments, dont les règles générales sont fixées dans les articles 208 à 210 du Code de la Santé (Loi n $\left.{ }^{\circ} 2011-002\right)^{12}$. Le dépôt est un centre de vente et de distribution de produits pharmaceutiques de première nécessité, tenu par un dépositaire devant être détenteur d'un baccalauréat et formés pendant deux mois au

Revue francophone sur la santé et les territoires, Les circulations en santé : des produits, des savoirs, des personnes en mouvement 
sein d'une pharmacie. L'autorisation d'exploitation des dépôts est délivrée à titre palliatif par le Ministère de la Santé à des non-pharmaciens, dans les localités où il n'y a pas d'officine pharmaceutique Ce dépôt est autorisé à vendre les 183 médicaments et consommables médicaux figurant sur la liste édifiée par le Ministère de la Santé. Les médicaments y sont disponibles sous différentes formes (comprimé, poudre, gélule, injections, gel, spray, sirop, etc.). Concernant la localisation géographique de ces dépôts, le Code de la Santé prévoit que l'implantation d'une pharmacie dans un périmètre inférieur à $10 \mathrm{~km}$ à un dépôt rend caduque toute autorisation antérieure délivrée au profit d'un dépôt de médicaments déjà fonctionnel. Le dépôt dispose alors d'un délai de trois mois pour fermer ses portes.

Il arrive que certains dépositaires ouvrent un ou plusieurs dépôts de médicaments « informels » qu'ils approvisionnent sous couvert de leur licence ${ }^{13}$ officielle et dont ils délèguent la gestion à un membre de leur famille. Ces dépôts « informels » peuvent être ouverts dans la même commune que le dépôt "mère ", ou à l'autre bout du pays. Ce mécanisme sera précisé dans la suite du texte.

\section{Organisation et fonctionnement du secteur informel à Madagascar}

16 Pour mieux comprendre le rôle des dépôts dans la circulation informelle de médicaments sur l'île et leurs mécanismes d'approvisionnement, il est nécessaire de décrire d'abord les différentes caractéristiques du secteur informel à Madagascar et plus spécifiquement à Antananarivo où se trouve le quartier d'Ambohipo, communément reconnu comme étant la référence nationale en matière de vente informelle de médicaments.

\section{Deux types de vendeurs informels à Ambohipo}

Le quartier Ambohipo, situé au sud-est de la capitale, abrite la cité universitaire et de nombreux commerces (boucheries, friperies, épiceries, quincailleries). Le quartier s'étend sur plusieurs kilomètres au nord et à l'est, mais la majeure partie des commerces de médicaments se trouve aux «trois chemins », un lieu-dit qui se situe au cœur du quartier d'Ambohipo. Il est particulièrement fréquenté par les étudiants de l'Université située à quelques centaines de mètres du quartier. Le quartier est bordé au nord par une officine pharmaceutique et au sud par un Centre de Santé de Base public, équipé d'une pharmacie. Deux dispensaires privés y exercent et trois docteurs privés y sont recensés au moment de notre enquête.

Pour des raisons historiques (Mattern, 2017) ${ }^{14}$, ce quartier est le nœud principal de circulation des réseaux informels, c'est là que se concentrent les boutiques et points de ventes exclusivement dédiés à la vente de médicaments informels. La vente informelle de médicaments s'y appuie fondamentalement sur les liens familiaux. Quatre grandes familles de l'ethnie Betsileo (entendu ici au sens de la parenté élargie - cousins, beauxfrères, ce que les interlocuteurs sur le terrain appellent la famille " lointaine " et la famille " proche ", ils font partie d'une même famille élargie au sens où ils sont tous les descendants d'un même ancêtre) se divisent l'espace du quartier d'Ambohipo, ces familles sont à la tête de réseaux de distribution de médicaments sur l'ensemble du pays, ces réseaux étant indépendants les uns des autres. Ces familles sont toutes 
originaires de la commune d'Imadiala située dans la région d'Amoron'I Mania, dans le district d'Ambositra situé à $250 \mathrm{~km}$ au sud de la capitale. L'ensemble des vendeurs rencontrés provient de cette zone géographique et les données collectées donnent à penser que l'ensemble des vendeurs de médicaments d'Ambohipo sont originaires de ce district.

Depuis l'émergence du marché informel du médicament en 1970, un groupe social d'acteurs issus de l'ethnie Betsileo en est à la tête. La particularité de ce marché ne réside pas tant dans la spécialisation du commerce de médicaments à Madagascar par un réseau circonscrit, à savoir les Betsileo, fait relativement classique et déjà traité dans d'autres contextes géographiques, à d'autres époques et pour d'autres types de marchandises (Agier, 1983 ; Ayimpam, 2013 ; Baxerres, 2014). L'intérêt ici réside dans le fait que depuis la mise en circulation des premiers médicaments informels au niveau de l'ile par un groupe d'étudiants Betsileo jusqu'à aujourd'hui, c'est ce groupe qui s'impose dans la gestion de ce commerce. Depuis près de 50 ans donc, le marché informel du médicament reste spécifiquement géré et maintenu par des individus issus d'un même groupe ethnique. La vente de médicaments est un motif de migration pour les vendeurs depuis le district d'Ambositra vers Antananarivo, au même titre que les autres " business » dans lesquels les commerçants sont investis.

20 L'argent du médicament a fait pousser les seuls bâtiments récents du quartier d'Ambohipo, construits de part et d'autre de la route en terre qui mène à l'Université. Ces bâtisses aux vitres teintées (des habitations pour la plupart) dénotent dans un paysage fait de petites habitations de fortune.

21 A Ambohipo, deux types de vendeurs de médicaments exercent leur pratique commerciale : les « petits vendeurs » et les " grossistes » ou " demi-gros ${ }^{15}$ selon les terminologies les plus fréquemment utilisées par ces acteurs eux-mêmes. En termes de volume de médicaments disponibles sur le marché informel, ce sont ces derniers qui représentent la part la plus importante, comme nous le décrivons plus loin. Outre ces deux catégories de vendeurs, deux acteurs rendent également possible la vente : les «fournisseurs » et les « intermédiaires ». Les «fournisseurs» constituent le lien entre les particuliers souhaitant revendre leurs médicaments (suite à une hospitalisation, un décès, un épisode de maladie) et les vendeurs informels, intéressés par l'achat de ces médicaments. Tandis que les « intermédiaires » (mpanera), sont les acteurs de première ligne. Leur rôle au sein du marché informel est double : ils orientent les fournisseurs vers des vendeurs qui pourraient être intéressés par leurs marchandises et ils constituent le relais entre les vendeurs et les clients qui ne connaissent pas de vendeurs en particulier, ou qui ne souhaitent pas se déplacer dans le quartier.

Les «petits vendeurs» et " grossistes " partagent une origine commune et appartiennent tous à l'ethnie Betsileo. Ces hommes et ces femmes sont âgés entre 20 et 55 ans. Les deux catégories de vendeurs se différencient par la quantité de médicaments dont ils disposent pour la vente : les « petits vendeurs » disposent moins de références que les " grossistes ». Ils se différencient également par leurs sources d'approvisionnement en médicaments et par les types de médicaments vendus (spécialités ou génériques).

23 Les modes d'approvisionnement des vendeurs informels d'Ambohipo sont pluriels. Chaque acteur de la vente dispose de ses propres filières d'approvisionnement en fonction des contacts qu'il détient au sein des grossistes pharmaceutiques formels, des 
réseaux à l'étranger, des réseaux de fournisseurs particuliers, de contacts au sein des pharmaciens d'officines ou de médecins dans les centres de santé publics.

\section{Formes et fonctionnement des commerces tenus par les " petits vendeurs"}

24 Les vendeurs enquêtés appartenant à cette catégorie pratiquent la vente de médicaments depuis une durée variant de deux à dix ans. Le démarrage de leurs activités commerciales correspond souvent au moment de leur arrivée à Antananarivo. Avant de se reconvertir à la vente de médicaments, une partie d'entre eux possédaient un petit commerce (épicerie principalement), d'autres étaient agriculteurs à Ambositra. Ces vendeurs justifient cette reconversion en raison des pratiques particulièrement lucratives de la vente de médicaments.

Derrière la dénomination " petits vendeurs " opèrent des catégories inégales de commerces quant à l'offre en produits pharmaceutiques proposée. L'ampleur de leur commerce peut varier fortement en fonction de la somme investie dans le commerce, des types de médicaments vendus et de la clientèle visée.

Si on décide de se spécialiser dans les génériques, les bénéfices seront plus petits mais le stock s'écoulera plus facilement. C'est comme pour les épiceries. Tandis que les spécialités rapporteront des bénéfices plus élevés mais ça se vend moins bien (Vendeuse $\mathrm{n}^{\circ} 10$ ).

Les petits vendeurs travaillent seuls. Dans une volonté de maximiser les bénéfices, leur commerce est géré individuellement ou par deux ou trois membres de la famille.

Enquêteur : Pourquoi vouloir travailler seule plutôt que de rester avec votre cousin [qui vous a formé à la vente de médicaments] ? Enquêtée : Les bénéfices sont plus élevés quand on est seul. Les Betsileo aiment la collectivité, surtout en cas de décès. Mais pour ce qui est du business, on est plutôt individuel (Vendeuse $n^{\circ} 11$ ).

Ces vendeurs passent une majeure partie de leur temps assis devant leur maison, à discuter avec les passants et d'autres commerçants, à la recherche de nouveaux clients. Selon ces vendeurs, il n'est pas difficile de reconnaitre un potentiel client : " souvent, il se promène et on voit bien qu'il cherche quelque chose " nous dit l'un deux. Le client potentiel est alors interpellé, et commence l'échange concernant les besoins en termes de médicaments.

Ces petits vendeurs travaillent principalement avec une clientèle fidélisée. Leurs points de vente ne sont pas visibles depuis la rue car les marchandises sont stockées dans des cartons ou dans des armoires, au domicile même des vendeurs. A l'instar des autres habitations du quartier et plus largement de la capitale, ces lieux de vie sont exigus et comportent au mieux deux pièces (salon et chambre), la cuisine se fait à l'aide d'un foyer à charbon de bois à l'extérieur de la maison. La pratique de vente de ces petits vendeurs est donc fortement imbriquée dans la vie familiale, les mères et les pères vendent leurs médicaments tout en continuant leurs activités quotidiennes.

Le nombre de références pharmaceutiques dont ils disposent dépend fortement des vendeurs et varient d'une dizaine à une cinquantaine. Les produits les plus vendus sont disponibles (en fonction de la saisonnalité des maladies) en quelques exemplaires mais il n'est pas rare que les vendeurs soient en rupture de stock. Dans le cas où un 
médicament demandé par un client fidélisé vient à manquer, le vendeur se déplace luimême auprès d'autres vendeurs.

31 Les petits vendeurs ne disposent pas de procédures de systématisation de leurs stocks de médicaments et de leurs ventes qui pourraient être par exemple des listes des prix et des médicaments disponibles, carnet listant les médicaments vendus, carnet par client «fidélisé ». Pour les médicaments vendus couramment, ils connaissent de mémoire le contenu de leur stock, ainsi que les prix. Chaque petit vendeur développe ses filières propres d'acquisition de médicaments. Leurs marchandises proviennent de différentes sources. L'une des sources d'approvisionnement de cette catégorie de vendeurs est constituée des fournisseurs.

\section{Les « fournisseurs » comme interface entre client et vendeur}

Ces fournisseurs proposent tous types de médicaments (spécialités et génériques) et sous différentes formes : boîte complète, plaquette dont une partie des comprimés ont été utilisés, etc. Ils passent à Ambohipo une à deux fois par semaine pour proposer aux vendeurs les médicaments dont ils disposent. Ces médicaments sont issus du secteur public et privé (hôpitaux, centres de santé, organismes de prise en charge de la santé des employés de certaines entreprises). Il arrive également que les vendeurs aient des accords avec des Malgaches qui dans le cadre de leur travail font des va et vient avec la France (du personnel de compagnies aériennes par exemple, ou qui ont de la famille en France). Les médicaments provenant de dons peuvent également finir dans les paniers des fournisseurs (détournement des dons destinés à des organisations caritatives). Certains petits vendeurs acquièrent une partie de leurs médicaments auprès des " grossistes ». Ce phénomène de revente constitue un des points d'articulation entre les circuits formels et informels.

\section{Les « grossistes » : principaux fournisseurs des dépôts de médicaments informels}

33 À Ambohipo, les marchandises des " grossistes » sont rangées dans des espaces réservés à la vente, des boutiques propres et simplement meublées (table et chaise pour la discussion avec le client, et bancs pour l'attente). A la différence des petits vendeurs qui entreposent leurs médicaments dans leur lieu de vie, les "grossistes » disposent d'un local attenant à leur domicile ou à un autre commerce dont ils sont propriétaires également. Un rideau peut être tiré devant les étagères afin d'en dissimuler le contenu.

$\mathrm{Au}$ contraire des petits vendeurs, les « grossistes » ont mis en place des procédures de systématisation de leurs ventes. Outre le système de recensement des ventes dans des cahiers, les vendeurs ont développé des systèmes de gestion de leur stock : renouvellement des stocks de médicaments dont la date de péremption est proche, achat de médicaments dont la date de validité est supérieure à deux ans, constitution de réserves en faible quantité pour les médicaments qui ne s'écoulent pas facilement, anticipation de l'approvisionnement en médicaments en fonction de la saisonnalité des maladies.

Les principaux clients de ces "grossistes " se situent aux quatre coins de l'ile. Les "grossistes » d'Ambohipo échangent principalement par téléphone avec les clients qui résident en province : quelqu'un est chargé par l'acheteur de récupérer la commande 
en main propre, la facture se règle alors directement entre les deux individus. Il arrive souvent que les colis de médicaments soient envoyés via des coopératives de taxibrousse. Dans ce cas, la transaction s'opère via les services de transfert d'argent et de paiement, accessibles via la téléphonie mobile (Orange Money, MVola, etc.). Nous avons observé des " grossistes " en train de préparer de gros cartons de médicaments à destination d'un point de vente informel en province. Ces acteurs nommés "grossistes" par les habitants d'Ambohipo, ne sont autres que des dépôts de médicaments « informels ».

\section{Interactions entre dépôts informels de médicaments et grossistes importateurs répartiteurs}

Le mécanisme d'approvisionnement des dépôts informels de médicament nécessite une certaine complaisance de la part des grossistes importateurs répartiteurs. Les extraits ci-dessous illustrent cette idée.

Certains grossistes [grossistes répartiteurs] aussi sont impliqués [dans la vente informelle de médicaments]. Ils ne sont pas directement impliqués dans le sens où ils vendent à des dépositaires [agrées] qui ensuite revendent sur le marché informel. Mais ils devraient avoir la puce à l'oreille car des petits dépositaires qui vendent pour 50 millions d'ariary de marchandises [plus de $14000 €$ ] dans une localité de 1000 habitants, ça parait clair ("Grossiste " ${ }^{\circ} 7$ ).

Enquêteur : «Tiana, vous ravitaillez des points de vente à Tana avec la patente de votre oncle, propriétaire d'un dépôt de médicaments dans une localité du sud est de Madagascar. Lydia de votre côté, vous ravitaillez des marchands ambulants, toujours avec les marchandises achetées auprès du grossiste répartiteur également grâce à la patente de votre oncle. Quand vous faites votre commande, le grossiste doit être étonné que pour une si petite localité, vous commandiez autant de médicaments ?» Enquêté : « Non il n'est pas étonné, le grossiste sait » (Vendeuses n ${ }^{\circ} 12$ et 13 ).

Comme l'indiquent ces deux extraits, les grossistes répartiteurs cités ici choisissent de fermer les yeux, sans que cela ne les mette à mal vis-à-vis de la règlementation pharmaceutique, car la personne en charge de la récupération des commandes dispose bien des autorisations requises. Ils ferment les yeux sur le montant de médicaments demandés, même s'il dépasse largement les besoins de la localité. En ce sens, certains grossistes répartiteurs contribuent de manière indirecte à l'approvisionnement $d u$ marché informel. Il y a donc une réelle intrication des secteurs formels et informels.

\section{Le rôle des dépôts de médicaments dans l'approvisionnement du secteur informel}

Les dépositaires interrogés à Ambohipo et Imadiala justifient l'ouverture de dépôts informels de médicaments (sous couvert de la licence d'un dépôt formel) par les problèmes d'écoulement de leur stock dans des localités où les populations ont un faible pouvoir achat et donc recourent assez peu aux dépôts de médicaments. Comme le suggère l'extrait d'entretien ci-dessous, la stratégie est alors d'ouvrir un autre dépôt, cette fois-ci informel, dans une zone où la clientèle serait plus nombreuse. L'exemple donné ci-dessous décrit l'ouverture d'un dépôt informel ouvert à Ambohipo.

Il y a souvent des plaintes de la part des pharmaciens envers les dépôts de médicaments. Ils disent que ce sont eux qui sont à l'origine du marché illicite. Ce 
n'est pas faux. Mais c'est difficile d'avoir un dépôt à la campagne, les gens n'ont pas d'argent pour acheter des médicaments. Les dépositaires ont des difficultés pour faire vivre leurs familles, donc ils ouvrent un point de vente à Tana (Membre de l'association Betsileo).

Concrètement, le chemin emprunté par les médicaments passe directement des grossistes répartiteurs de la capitale vers les dépôts informels ou " grossistes » s'ils sont situés à Antananarivo. C'est eux qui ensuite s'occupent d'acheminer les médicaments dans le dépôt formel, situé en milieu rural. Nos observations au sein de ces "grossistes " à Ambohipo ont confirmé cette hypothèse : nous y avons régulièrement vu des cartons affublés du nom d'un grossiste répartiteur, envoyés par taxi-brousse vers d'autres points de vente.

41 Afin de saisir finement les mécanismes d'approvisionnement de ces dépôts de médicaments, prenons les exemples emblématiques d'Aimé, propriétaire de dépôts (formel et informel) situés en milieu rural et aux quatre coins du pays et de Hasina, « grossiste » à Ambohipo.

\section{Aimé, 48 ans, businessman reconnu à Imadiala}

Aimé est un homme de 48 ans. Il est marié et a cinq enfants. Il a commencé par travailler dans le secteur " formel », dans "la légalité », comme il aimait le dire. Mais " même si je faisais bien mon travail, et dans la légalité, je me faisais avoir par d'autres, je ne parvenais donc pas à gagner de l'argent ». Encouragé par des membres de sa famille déjà engagés dans la vente informelle de médicaments, il a commencé à travailler dans " l'illicite ». Il possède trois maisons : une à Antananarivo qu'il loue, une autre en construction dans la commune d'Imadiala, «C'est une maison faite selon les plans de France ", nous dit-il, et une dernière à Fianarantsoa. Notre entretien se déroule dans sa grande maison à trois étages en cours de construction dans la commune d'Imadiala, elle comporte quinze chambres.

Son cousin et son frère font partie des premiers vendeurs ayant monté leur " business » à Ambohipo en 1980, sa belle-sœur est propriétaire d'un dépôt de médicaments à l'ouest de Madagascar et d'une boutique à Antananarivo. Aimé est le propriétaire de plusieurs dépôts de médicament, dont un qui se situe à $12 \mathrm{~km}$ de son domicile. Il dispose d'une licence prouvant qu'il en est le propriétaire et pour pouvoir se consacrer à d'autres activités économiques, il a laissé la gestion du dépôt aux soins de sa nièce Mbola qui nous dit :

Enquêteur : Quel âge avez-vous et depuis combien de temps travaillez-vous ici ? Enquêtée : J'ai 19 ans. Ce dépôt est ouvert depuis 2005 mais le premier proprio est décédé, il a légué le fond à son grand père, puis lui à son fils. Ce fils est maintenant le propriétaire et il est le mari de ma sœur. Enquêteur : C'est vous qui avez choisi de travailler ici ? Enquêtée : J'ai été désignée. Je n'aime pas trop mon boulot mais je suis obligée de le faire pour aider ma sœur. Mes frères et sœurs sont déjà mariés. Je ne gagne pas de salaire fixe mais quand j'ai besoin d'argent ma sœur m'en donne. Enquêteur : Vous vendez plutôt des médicaments de spécialité et des génériques ? Enquêtée : Je ne connais pas la différence (Mbola, dépositaire Ambositra).

Mbola a donc été désignée par son oncle pour tenir le dépôt. Elle nous dira plus tard qu'elle souhaite poursuivre des études à Fianarantsoa et que pour cela, elle sera aidée par son oncle, raison pour laquelle elle a accepté de gérer son dépôt. 
Pour l'approvisionnement de ses dépôts, Aimé achète les médicaments auprès d'un grossiste importateur répartiteur à Antananarivo sous couvert de la licence dont il dispose pour son dépôt formel. Il revend ensuite à plusieurs types de clients: marchands ambulants, "grossistes ", épiciers. Il réalise des ventes de médicaments à domicile et tient également un étale de médicaments sur le marché communal dans la région d'Ambositra. Ce marché ravitaille tous les marchands ambulants et tous les petits points de vente informelle de médicaments de la région. Selon lui, ces pratiques procurent un maximum de profits. "J'étais cultivateur, je mangeais des brèdes [sorte d'épinard] du matin au soir. Maintenant je peux acheter du poisson, de la viande, j'ai même une maison avec électricité » nous dit-il.

\section{Hasina, 25 ans, propriétaire d'une épicerie et d'un dépôt informel à Ambohipo}

En 2016, Hasina a 2 enfants et vit depuis plusieurs années à Ambohipo. A côté de son travail de revendeur, il était engagé dans un commerce d'exportation d'haricots vers l'île Maurice. En juin 2015, ses activités commerciales ont cessé. En 2006 il a arrêté ses études. En 2009 il ouvre un dépôt de médicaments à Fianarantsoa. Son souhait initial était d'ouvrir une pharmacie mais les coûts liés à l'investissement dans un fonds de commerce étaient trop importants, il a donc décidé d'ouvrir un dépôt de médicaments. Aujourd'hui, c'est sa famille qui s'occupe de son dépôt légal à Fianarantsoa, à 290 kilomètres dans le sud de la capitale. C'est en venant à la capitale pour régler les papiers pour son dépôt qu'il constate que le marché informel du médicament y était florissant. Il ouvre alors d'abord une épicerie (pour justifier les va-et-vient dans ses locaux et pour servir de " couverture » à son commerce informel de médicaments), puis un dépôt informel, "puisqu'il avait de toute façon les papiers du dépôt légal de Fianarantsoa ».

"J'ai la photocopie des papiers de mon dépôt à Fianarantsoa, alors en cas de rupture de stock même dans mon dépôt de Tana, il suffit que j'aille voir le grossiste, sans passer par Fianarantsoa. Le seul dépôt légal que j'ai est à Fianarantsoa ». (Hasina)

Le mécanisme décrit dans ces deux récits est donc simple : un dépôt de médicaments est ouvert avec autorisation d'exploitation délivrée par le Ministère de la Santé Publique. Le Code de la Santé prévoit que le dépositaire s'approvisionne auprès d'un pharmacien. Dans la pratique, les dépositaires se procurent leurs médicaments directement auprès des grossistes formels. Au moment de passer commande auprès d'un grossiste répartiteur, le dépositaire gonfle sa liste de médicaments, il commandera donc par exemple un nombre trois fois plus élevé de comprimés de paracétamols qu'il n'en faut pour sa localité. A Antananarivo, un relais (souvent un membre de la famille) va chercher la commande auprès du grossiste répartiteur muni de l'autorisation d'exploitation en bonne et due forme du dépôt formel. Il ponctionne ensuite une partie des marchandises pour son point de vente informel et fait acheminer le reste des médicaments vers le dépôt formel. Les propriétaires et gérants de dépôts de médicaments sont souvent membres d'une même famille. Comme nous le voyons dans le récit de Aimé, les points de revente peuvent être multiples. Les grossistes importateurs répartiteurs et ces personnes " relais » chargées de récupérer la commande pour les dépôts formels de médicaments travaillent donc de concert, les uns 
de manière indirecte, les autres de manière directe pour l'approvisionnement des points de ventes informels.

La description de ce mécanisme d'approvisionnement démontre que les médicaments vendus par les dépôts informels de médicaments ou "grossistes" proviennent majoritairement du secteur formel, essentiellement privé.

\section{Controverses autour du rôle des dépôts de médicaments}

50 Parmi les différents acteurs du système pharmaceutique malgache, les dépôts sont au cœur de nombreuses controverses. La Direction de la Pharmacie, des Laboratoires et de la Médecine Traditionnelle (DPLMT) rencontre de nombreux problèmes dans la gestion et le contrôle de ces dépôts formels. Nous avons noté plusieurs écarts entre le cadre règlementaire régissant les dépôts de médicaments et les pratiques réelles.

$51 \mathrm{Au}$ moment de leur création, il était prévu que les dépôts soient mis sous tutelle de pharmaciens, garants des bonnes pratiques des dépositaires. Les textes prévoient que le dépositaire se forme pendant 3 mois auprès de son pharmacien "garant » et c'est également auprès de lui qu'il doit s'approvisionner en médicaments. Il incombe au dépositaire formé de s'occuper ensuite de la gestion de son dépôt. En réalité, la durée de formation du dépositaire est souvent raccourcie. Et comme décrit plus haut, le dépositaire s'approvisionne dans la pratique directement auprès des grossistes importateurs répartiteurs de la capitale. Comme illustré plus haut par le cas de Mbola, la nièce d'Aimé, les personnes en charge de la gestion quotidienne du dépôt ne sont pas forcément celles qui ont suivi la formation. Elles sont désignées par la famille et peuvent bénéficier en échange de leur implication d'avantages, souvent en termes d'appui financier (par exemple pour une future scolarisation). Elles bénéficient d'une connaissance limitée en matière de médicaments.

52 Ces écarts de pratiques font émerger des tensions entre les pharmaciens et les dépositaires.

\section{Les tensions entre pharmaciens et dépositaires}

53 Le débat qui oppose pharmaciens et dépositaires s'articule plus particulièrement autour deux questions. La première concerne le rôle que s'attribuent les dépositaires qui, selon nos interlocuteurs (pharmaciens, acteurs de la DPLMT) et selon nos observations et entrevues dépassent largement le cadre d'activités fixé au départ à deux niveaux : l'extension de la vente de médicaments par les dépôts au-delà de la liste prévue d'une part (183 médicaments et consommables médicaux) et l'« imitation » du rôle de pharmacien par le dépositaire, à savoir la qualité de dispensateur de produits pharmaceutiques mais également de conseiller pour l'utilisation des médicaments (dosage, équivalence).

54 Tout d'abord, la vente de médicaments qui ne figurent pas sur la liste des médicaments autorisés est pratique courante au sein des dépôts formels et informels.

"Ces dépôts ne sont pas tenus par des pharmaciens mais par des dépositaires

formés pendant 3 mois en officine, ils sont donc autorisés à dispenser des médicaments. Ils ont une liste de médicaments qu'ils peuvent distribuer, mais « les

Revue francophone sur la santé et les territoires, Les circulations en santé : des produits, des savoirs, des personnes en mouvement 
plus dangereux " sont réservés pour la vente en pharmacie. Mais en pratique, il arrive que les dépôts donnent des médicaments qui ne sont pas sur la liste car il faut bien que ces médicaments soient disponibles également au niveau des campagnes, dans ce cas précisément il y a un écart entre la théorie, la loi et la réalité (Un grossiste répartiteur de la capitale).

Comme le suggère cet extrait d'entretien, des médicaments non inclus dans la liste $\mathrm{y}$ sont vendus. Pour se justifier, les dépositaires avancent que dans un contexte d'absence d'autres offres de produits pharmaceutiques, il est normal qu'ils puissent dispenser des médicaments dont les populations ont besoin même s'ils ne figurent pas sur leur liste (ont été cités notamment certains types d'antibiotiques). A ce sujet, dépositaires et acteurs de la Direction de la Pharmacie des Laboratoires et de la Médecine Traditionnelle se rejoignent, tous deux conscients des difficultés d'accès aux médicaments dans les zones enclavées. Une norme officieuse s'instaure donc : les dépôts étendent la liste de médicaments autorisés à la vente à une série de médicaments qu'ils estiment utiles aux populations résidant dans leurs localités.

Le second point concerne la limitation du rôle du dispensateur à celui de « vendeur de médicaments ». A l'opposé, le pharmacien est habilité à dispenser des médicaments et des conseils, ainsi qu'à exécuter des préparations officinales. Les relations entre pharmaciens et dépositaires sont tendues à ce sujet : les premiers se targuent d'avoir fait 8 ans d'études, ils sont habilités à vendre leurs produits, tout en conseillant leur clientèle. Les seconds sont considérés comme de simples commerçants qui endossent trop souvent un rôle de conseiller. Les pharmaciens récusent la possibilité pour les dépositaires de dépasser leur rôle de vendeur et d'embrasser ce rôle de conseiller.

: Vous donnez des conseils en même temps que la vente ? Enquêté : Nous expliquons, renforçons les explications données par le docteur. Parce que les gens d'ici n'aiment pas les docteurs, ils en ont peur, ils ne comprennent pas bien ce qu'ils leur disent. Alors on donne des messages plus clairs. Puis on donne des conseils par exemple de ne pas manger de fruits (letchis), des glaces à l'eau parce que ça donne des maux de ventre. On conseille aussi d'aller souvent chez le docteur. Avant que les dépôts ne soient ouverts, les gens achetaient beaucoup leurs médicaments aux marchands ambulants (dépôt formel de médicament à Ifanadiana).

Un autre point échappe au contrôle des autorités règlementaires. Un arrêté ministériel (n¹1092/2016) prévoit que le local destiné à la vente de consommables médicaux doit être exclusivement réservé à cet usage. En pratique, il n'est pas rare de voir des dépositaires proposer pour la vente d'autres types de marchandises : des denrées alimentaires par exemple. Les arguments avancés par ces dépositaires s'appuient sur la concurrence établie entre les différents dépôts au sein d'une même localité alors même que la demande en produits pharmaceutiques est moindre. dépôts, ces lieux de vente sont pointés du doigt par les acteurs institutionnels de distribution du médicament et identifiés comme étant le point de départ pour la fuite des médicaments du secteur formel vers l'informel comme nous l'avons démontré plus haut.

Dans les faits, il est difficile de reconnaître un dépôt formel d'un informel, la seule différence étant la possession de documents autorisant l'ouverture régulière du dépôt. L'implantation des dépôts de vente comme solution palliative était au départ une réponse temporaire, mais elle tend aujourd'hui à se pérenniser. En raison de 
nombreuses difficultés liées à la gestion de ces points de vente, la DPLMT ne délivre plus depuis 2015 d'autorisation d'implantations de nouveaux dépôts.

\section{Conclusion : un flou des frontières entre le formel et l'informel}

61 La présentation du système pharmaceutique malgache et l'analyse du rôle incontournable des dépôts de médicaments dans la circulation des médicaments à Madagascar nous invite à aborder un point de discussion majeur qui concerne l'étanchéité des catégories dites «formelles» et «informelles» de distribution pharmaceutique. En effet, comme nous l'avons vu, dans le secteur de la distribution de médicaments, la frontière entre le formel et l'informel est floue. A ce propos, un grossiste nous dit ceci: "Le pharmacien vend sans ordonnance, le médecin vend des médicaments et le patient achète n'importe où ses médicaments, il est temps que Madagascar s'allonge sur un divan et fasse sa psychanalyse ". A travers l'énonciation de ces faits, ce grossiste nous rappelle l'existence à Madagascar d'un écart important entre les normes officielles (celles qui régulent l'organisation géographique des espaces de vente par exemple, ou encore les normes visant à autoriser la vente de médicaments par certains acteurs, relevant exclusivement d'une liste) et les normes pratiques (Olivier de Sardan, 2001b). Les comportements des différents acteurs de distribution renvoient à des normes réelles et ne constituent pas un "simple » écart de la norme officielle ${ }^{16}$. Si Madagascar dispose d'une autorité de réglementation autonome, d'un cadre légal clair et de documents pharmaceutiques de référence nationale ${ }^{17}$, la distribution pharmaceutique est caractérisée par d'importantes faiblesses, qui se cristallisent notamment autour des dépôts de médicaments (Andriamparany, 2017).

Ce constat est vrai dans plusieurs domaines ayant trait à la distribution pharmaceutique. Les exemples de ces écarts sont nombreux. Par exemple, la loi légiférant le nombre de dépôts dans une localité ou encore celle qui prévoit une fermeture directe du dépôt au moment de l'installation d'une pharmacie dans un périmètre précis $(10 \mathrm{~km})$ sont dans les faits, rarement appliquées. Certains pharmaciens déplorent le maintien de l'autorisation d'exercer de dépôts installés en deçà du périmètre autorisé. Dans la pratique nous avons également observé des pharmacies dont la gestion était confiée à un non-pharmacien, celui-ci ayant racheté ou loué le diplôme du premier. Un autre exemple décrit plus haut se rapporte plutôt aux dépôts des médicaments qui au vu et au su des autorités de règlementation dispensent des médicaments qui ne figurent pourtant pas dans la liste des médicaments autorisés. Certains grossistes vendent des références qui ne disposent pas ou plus d'AMM, d'autres vont à l'encontre de la loi leur interdisant de vendre au détail. En bref, ces exemples illustrent l'idée qu'à Madagascar comme dans d'autres contextes géographiques, l'existence d'un cadre règlementaire n'assure aucunement son application.

Les dépôts de médicaments constituent une catégorie de points de vente dont les caractéristiques décrites ici semblent être inédites dans le paysage international de la vente informelle de médicaments. En effet les dépôts de médicaments détiennent une place importante dans l'approvisionnement du circuit informel à Madagascar et parviennent, par des mécanismes simples à alimenter un nombre important de points 
de vente informels. Ces dépôts constituent le point d'articulation majeur entre les secteurs formels et informels de distribution pharmaceutique.

L'existence de cet acteur démontre l'extrême flexibilité dont fait preuve le marché informel du médicament à Madagascar. L'informalité parvient à s'engouffrer dans les failles d'un système qui évolue, rendant les médicaments disponibles à la fois aux ménages ruraux, aux urbains et aux différents groupes sociaux. Comme démontré plus haut, la pratique de vente informelle de médicaments permet l'émancipation d'un groupe social. Par ailleurs, le marché informel du médicament facilite l'accès aux médicaments pour les populations géographiquement isolées, pour les groupes populaires (C. Mattern, 2018) ou même pour les sujets de catégorie moyenne et aisée à la recherche de médicaments qui ne sont plus disponibles dans les secteurs formels ou qui présentent une charge financière trop lourde. Mais elle pose une double question : qu'en est-il de l'impact sur la santé publique ? Et enfin, qu'est-ce que cette pratique d'économie populaire suggère des horizons politiques et économiques dans lesquels elle se développe (Sarria Icaza et Tiria, $2005: 228$ ) ?

\section{BIBLIOGRAPHIE}

AGIER M., 1983, Commerce et sociabilité. Les négociants soudanais du quartier zongo de Lomé (Togo), Editions de l'office de la Recherche Scientifique et Techinique Outre-Mer - Collection Mémoires $\mathrm{n}^{\circ} 99,311 \mathrm{p}$.

ANDRIAMPARANY T.A., 2017, Accès aux médicaments essentiels de qualité au niveau des soins de santé primaires : analyse du système pharmaceutique de Madagascar., Thèse de doctorat, Anvers, Institute of tropical medicine, $65 \mathrm{p}$.

AYIMPAM S., 2014, Economie de la débrouille à Kinshasa. Informalité, commerce et réseaux sociaux, Paris, Editions Karthala, 332 p.

BAXERRES C., 2014, Du médicament informel au médicament libéralisé. Une anthropologie du médicament pharmaceutique au Bénin, Paris, Edition des archives contemporaines, $313 \mathrm{p}$.

BAXERRES C., HESRAN J.-Y. LE, 2011, « Where do pharmaceuticals on the market originate? An analysis of the informal drug supply in Cotonou, Benin », Social Science and Medecine, 73, p. 1249-1256.

BLOOM G., STANDING H., LUCAS H., BHUIYA A., OLADEPO O., PETERS D.H., 2011, « Making health markets work better for poor people: the case of informal providers », Health policy and planning, 26, p. 45-52.

BOURDIER F., Man B., Res P., 2014, La circulation non contrôlée des médicaments en Asie du SudEst et au Cambodge, L'espace politique, 24-3.

DOMINIQUE J.V., 2016, « Une sociologie de l'élite militaire : la cas des militaires malgaches formés en ex-URSS dans les années 80 », 4ème rencontre des études africaines en France, 5-6-7 juillet. 
MATTERN C., 2017, « Le marché informel du médicament à Madagascar : une revanche populaire », Thèse présentée en vue de l'obtention du grade de docteur en sciences politiques et sociales de l'Université catholique de Louvain, $421 \mathrm{p}$.

MATTERN C., 2019, «J'ai peur quand je fais le docteur pour mes enfants » : l'automédication au cœur des pratiques de soins des jeunes enfants à Antananarivo, Madagascar ", Anthropologie \& Santé [En ligne], 18 | 2019, URL : http://journals.openedition.org/anthropologiesante/4115 ; DOI : 10.4000/anthropologiesante.4115

MESTRE C., 2014, Maladies et violences ordinaires dans un hôpital malgache, Paris, L"Harmattan, 162 p.

NSARHAZA K., 1997, « comprendre le secteur informel de la santé (SIS). Concepts, techniques de collecte de données et structure pour l'analyse, Document technique Organisation mondiale de la santé, Série Macroéconomie, santé et développement, 31.

SUDHINARASET M., INGRAM M., LOFTHOUSE H.K., MONTAGU D., 2013, « What Is the Role of Informal Healthcare Providers in Developing Countries? A Systematic Review », Plos One, 8, 2.

OLIVIER DE SARDAN J., 2001b, « La sage-femme et le douanier. Cultures professionnelles locales et culture bureaucratique privatisée en Afrique de l"Ouest ", dans RAFFINOT M., ROUBAUD F. (dirs.), Les fonctionnaires du Sud entre deux eaux : sacrifiés ou protégés? (Autrepart), Editions de l"Aube, IRD, p. 61-73.

QUET M, PORDIE M, BOCHATON A, CHANTAVANICH S, KIATYING-ANGSULEE N, LAMY M, VUNGSIRIPHISAL P, 2018. Regulation Multiple: Pharmaceutical Trajectories and Modes of Control in the ASEAN, Science Technology and Society, SAGE Publications, 2018, DOI:

$10.1177 / 0971721818762935$

SARRIA ICAZA A.M., TIRIBA L., 2005, « Economie populaire », dans LAVILLE J., CATTANI A.D.

(dirs.), Dictionnaire de l'Autre économie, Paris, Desclée de Brouwer, p. 217-223.

\section{NOTES}

1. La dénomination " produits en santé " englobe tous les produits participant à l'obtention et au maintien d'un état de bien-être physique, mental et social (OMS, 1946).

2. Le quartier d'Ambohipo est situé dans le sud-est de la capitale, point de départ à partir duquel sont approvisionnés les différents réseaux de vente informelle de médicament à Madagascar. Il fera l'objet d'une description plus détaillée plus bas.

3. Les marchés permanents de Petite vitesse (Isotry), d'Analakely, de Pochard, d'Isotry, d'Ambodon'Isotry et du Coum (67 Ha), et les marchés hebdomadaires de Mahamasina (le jeudi) et d'Andrahavoahangy (le mercredi).

4. La SALAMA, établissement public à caractère industriel et commercial, fait partie de l'ACAME, soit l'association Africaine des Centrales d'achat de Médicaments Essentiels.

5. On entend par médicament essentiel les médicaments mentionnés dans la liste nationale des médicaments essentiels en vigueur, ou en son absence celle de l'OMS http://apps.who.int/medicinedocs/pdf/s2224f/s2224f.pdf

6. On entend par médicament générique toute copie essentiellement similaire à un médicament original, qui n'est plus protégé par un brevet d'exploitation. Il est 
commercialisé sous dénomination commune internationale (DCI) et/ou sous une dénomination spéciale http://apps.who.int/medicinedocs/pdf/s2224f/s2224f.pdf

7. Les PhaGeCom ont été implantées en 1998 par le Ministère de la Santé en vue de la généralisation de l'initiative de Bamako.

8. Le secteur privé à but non lucratif (confessionnel, interentreprises, ONG) s'approvisionne soit auprès de la centrale d'achat SALAMA, soit auprès de donateurs étrangers, pour la fourniture des intrants en santé destinés aux cliniques et centres de santé.

9. Durant la « Révolution socialiste » (1975-1992) sous le président Didier Ratsiraka, Madagascar entretenait des relations privilégiées avec les pays de l'ex URSS, constituant une opportunité de formation pour les Malgaches aisés (Dominique, 2016).

10. Calculé sur la base de l'estimation du nombre de la population 2014.

11. C'est-à-dire que les médicaments sont contenus dans un emballage pharmaceutique de deux formes : la forme "primaire " à savoir l'emballage pharmaceutique qui offre l'étanchéité et la forme de conditionnement "secondaire » à savoir l'emballage qui n'est pas en contact direct avec le médicament et inclut de manière générale les cartons imprimés ou non, les étiquettes ou notices. https://www.iso.org/obp/ui/fr/\#iso:std:iso: 15378:ed-4:v1:en:cd\%202\%20:v1\%20

12. Livre III titre V et chapitre premier du Code de la Santé.

13. Les personnes interviewées utilisaient fréquemment le terme "patente» pour référer à ces licences et autorisations d'exploitation. Ces trois terminologies seront donc utilisées ici.

14. Voir ma thèse pour plus d'informations

15. Terminologie utilisée par les vendeurs d'Ambohipo. Le terme " grossiste » ou " demi-gros " réfère aux vendeurs qui disposent d'une grande variété de médicaments. Les guillemets permettront tout au long du texte de les différencier des grossistes importateurs répartiteurs.

16. JPO de Sardan «La corruption quotidienne en Afrique de l'Ouest» https:// www.cairn.info/revue-politique-africaine-2001-3-page-8.htm «Les normes officielles ou assumées publiquement (elles-mêmes plurielles) et normes "réelles", relevant de cultures professionnelles locales, d'habitudes bureaucratiques, d'usages et de routines, de combines acceptées et de savoirs pratiques, de tours de mains et autres «coping stratégies " " .

17. WHO. Assessment of Medicines Regulatory Systems in Sub-Saharan African Countries. An overview of findings from 26 Assessment Reports [Internet]. World Health Organization. 2010 [cited 2017 Jun 6]. Available from: http://apps.who.int/ medicinedocs/documents/s17577en/s17577en.pdf

\section{RÉSUMÉS}

Résumé :

Revue francophone sur la santé et les territoires, Les circulations en santé : des produits, des savoirs, des personnes en mouvement 
Le présent article rend compte du rôle des dépôts de médicaments dans l'approvisionnement du secteur pharmaceutique informel à Madagascar. Les dépôts constituent un acteur incontournable dans la distribution pharmaceutique à Madagascar, ils ont été mis en place en 2011 pour pallier le manque de pharmaciens sur l'île ainsi que pour répondre aux problèmes d'accessibilités des populations aux médicaments essentiels. Partant de l'analyse de l'organisation de la distribution de médicaments à Madagascar (secteur privé, public et informel), nous y décrivons les caractéristiques, mécanismes de fonctionnement et modalités d'approvisionnement des dépôts de médicaments. Cet article se base sur les résultats de ma thèse, dont les données ont été collectées par trois biais : entretiens semi-directifs, observations directes et relevés des points de ventes informels. Une centaine d'entretiens semi-directifs a été réalisé entre 2013 et 2017 à Madagascar auprès d'acteurs issus des différents secteurs de distribution pharmaceutique.

\section{INDEX}

Mots-clés : Anthropologie, circulation, circuits informels, système pharmaceutique

Index géographique : Madagascar

\section{AUTEUR}

\section{CHIARELLA MATTERN}

Membre associée du CEPED (Institut de Recherche pour le Développement, Université de Paris, INSERM) et du LAAP (Laboratoire d'anthropologie prospective, Université Catholique de Louvain Belgique), chercheure responsable du service Santé \& Sciences Sociales, Unité Épidémiologie et de Recherche Clinique, Institut Pasteur de Madagascar, Antananarivo, Madagascar 